\title{
ESRGAN+ : FURTHER IMPROVING ENHANCED SUPER-RESOLUTION GENERATIVE ADVERSARIAL NETWORK
}

\author{
Nathanaël Carraz Rakotonirina, Andry Rasoanaivo \\ Laboratoire d'Informatique et Mathématiques, Université d'Antananarivo, Madagascar
}

\begin{abstract}
Enhanced Super-Resolution Generative Adversarial Network (ESRGAN) is a perceptual-driven approach for single image super-resolution that is able to produce photorealistic images. Despite the visual quality of these generated images, there is still room for improvement. In this fashion, the model is extended to further improve the perceptual quality of the images. We have designed a network architecture with a novel basic block to replace the one used by the original ESRGAN. Moreover, we introduce noise inputs to the generator network in order to exploit stochastic variation. The resulting images present more realistic textures. The code is available at https: / / github.com/ncarraz/ESRGANplus.
\end{abstract}

Index Terms - Super-resolution, Generative adversarial network

\section{INTRODUCTION}

Super-resolution (SR) is the task of generating a highresolution (HR) image using low-resolution (LR) ones. When only one LR image is used, it is commonly called Single Image Super-Resolution (SISR). The target of such task used to be the minimization of the mean squared error (MSE) between the generated image and the original one. This results in maximizing the peak signal-to-ratio (PSNR) which is a standard measure for SISR. However, PSNR-oriented approaches do not generate perceptually good images [1]. Perceptual-oriented methods were then proposed. SuperResolution Generative Adversarial Network (SRGAN) [1] uses both perceptual loss [2, 3] and generative adversarial networks (GANs) [4] to produce images residing in the manifold of natural images. Enhanced Super-Resolution Generative Adversarial Network (ESRGAN) [5] improves SRGAN by introducing an architecture composed of Residual-inResidual Dense Blocks (RRDB) without Batch Normalization (BN) [6] layers. Besides, relativistic average GAN (RaGAN) [7] was used as the discriminator and the features were used before activation.

We aim to further improve the perceptual quality of the images generated by ESRGAN. First, we propose a new block called Residual-in-Residual Dense Residual Block (RRDRB) which has higher capacity than ESRGAN's RRDB block.
Second, we introduce noise inputs in the network as in [8] in order to benefit from stochastic variation.

\section{RELATED WORK}

The main approaches to SISR can be divided into three distinct categories: interpolation-based methods, reconstructionbased methods and learning-based methods [9]. Approaches based on deep learning have further surpassed the two former methods as well as simple learning-based methods.

The very first deep learning-based approach, proposed by Dong et al. [10, 11], is SRCNN. It makes use of convolutional neural networks in an end-to-end manner. Though the network is shallow, it outsmarted previous techniques as far as the SISR task is concerned. Kim et al. [12] introduce a deeper model called VDSR. With a similar performance, DRCN [13] exploits deep recursive networks by combining intermediary results. SRResNet [1] and DRRN [14] make use of residual units. EDSR [15] along with MDSR, its multiple scale factors version, are the state-of-the-art methods for PSNR-based super-resolution. Residual dense networks were used in SRDenseNet and Memnet [16].

In order to focus more on the visual quality of generated images, a perceptual loss closer to perceptual quality is proposed. SRGAN which is based on GANs uses this perceptual loss along with adversarial loss to produce photorealistic images. These images are visually more convincing despite having lower score on standard quantitative measure like PSNR and structural similarity (SSIM). EnhanceNet [17] is also based on GANs but uses a different architecture. ESRGAN as its name implies enhances SRGAN. It introduced a new block with a higher capacity named RRDB. Besides BN layers were removed, residual scaling [18] and smaller initialization were used to facilitate training a very deep network. The discriminator uses relativistic average GAN, which learns to evalate "whether one image is more realistic than the other" rather than "whether one image is real or fake". Furthermore, in the perceptual loss, the VGG features are taken before activation rather than after as in SRGAN. There is still a gap between ground-truth images and images generated by ESRGAN. The present work aims to further close this gap. 


\section{METHOD}

\subsection{Network architecture}

ESRGAN's basic block allows the network to be easier to train and have a very high capacity. The overall architecture of ESRGAN is maintained as depicted in Figure 1 except for the Dense block which is replaced by our new block.

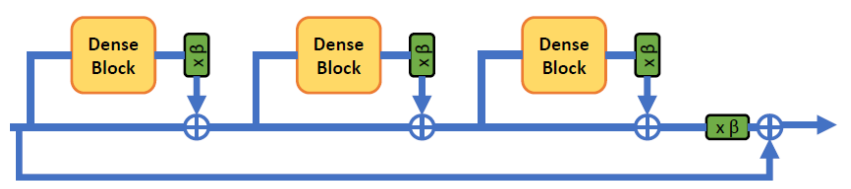

Fig. 1: The basic block used in ESRGAN called Residual in Residual Dense Block (RRDB).

The novel block we propose results in greater capacity. RRDB has a residual-in-residual structure with Dense blocks [15] in the main path. We add an additional level of residual learning inside the Dense blocks as presented in Figure 2 to augment the network capacity without increasing its complexity. A residual is then added every two layers in each Dense block. The visual quality of the generated images using the new block is substantially superior to that of the simple Dense block. As described in [19], ResNet enables to re-use features while DenseNet enables to find new features. This new architecture then benefits from both feature exploitation and exploration resulting in images of superior perceptual quality. We name ESRGAN+ the model using this new architecture.

\subsection{Noise inputs}

Adding noise to the generator was recently used in human faces generation [8] which also heavily relies on GANs. However, it was never applied to super-resolution. In order to have stochastic detail, noise inputs are introduced in the generator's architecture. Gaussian noise is added to the output of each residual dense block along with learned per-feature scaling factors, as illustrated in Figure 3 .

Stochastic variation randomizes only certain local aspects of the generated images without changing our global perception of the images [8]. The effects of the noise inputs are very localized leaving intact the general structure and the higher level information of the images. The network does not need to generate spatially-varying pseudorandom numbers when that is required. Consequently, the network capacity that would have been wasted for that task can be efficiently used to give finer-details in the high-level aspects. The model using both the new block and the noise inputs is called nESRGAN+.

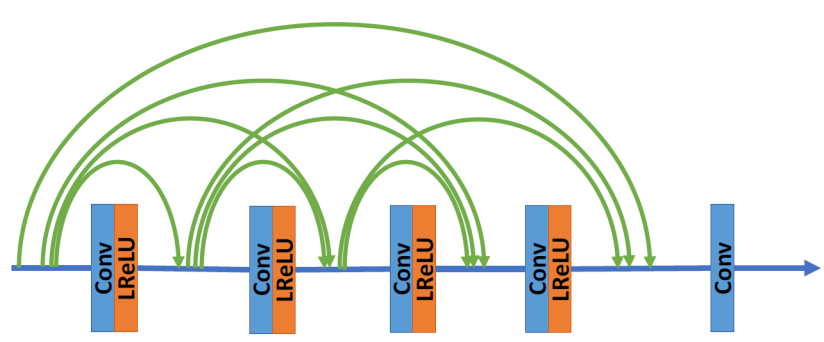

(a) Dense block

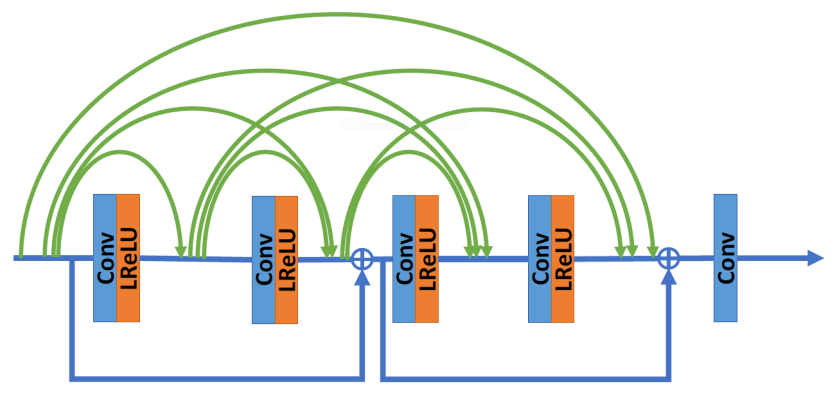

(b) Residual Dense block

Fig. 2: Top: Dense block is the main path used in ESRGAN's RRDB. Bottom: Residuals are added every two layer in the Dense block.

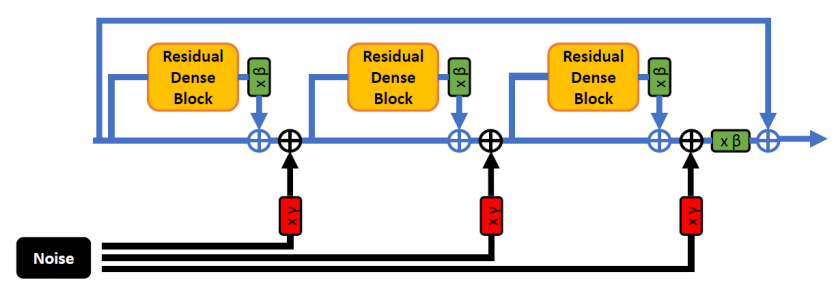

Fig. 3: Gaussian noise is added after each residual along with a learned scaling-factor.

\section{EXPERIMENTS}

\subsection{Data}

The used training set is DIV2K [20]. It is a dataset of $2 \mathrm{~K}$ resolution images adequate for the task of SR. Originally, there are only 800 images in the DIV2K dataset. As in ESRGAN, data augmentation is performed through random horizontal flips and rotations. The benchmark datasets used for evaluation are BSD100 [21], Urban100 [22], OST300 [23], Set5 [24], Set14 [25] and the PIRM datasets [26].

\subsection{Training details and parameters}

The LR images are obtained by downsampling the HR images using bicubic kernel with a scaling factor of $\mathrm{x} 4$. We maintain all the training parameters of the original ESRGAN. We crop $128 \times 128 \mathrm{HR}$ sub images. The size of the mini-batch is 16 . 


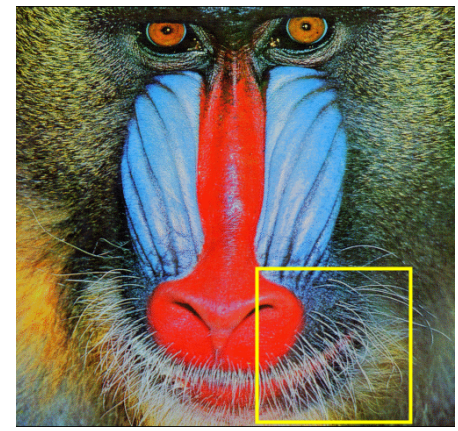

baboon from Set14

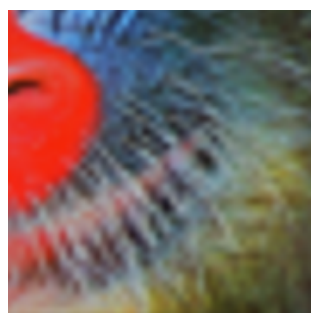

Bicubic

(22.43/6.77)

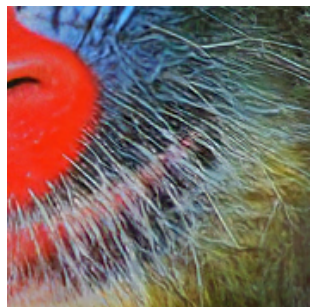

ESRGAN

(20.32/1.99)

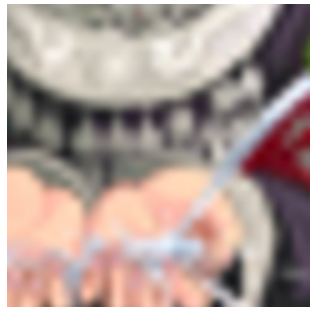

Bicubic

(21/6.73)

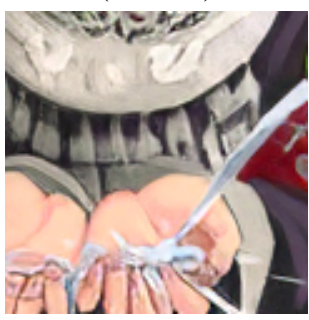

ESRGAN

(18.87/2.27)

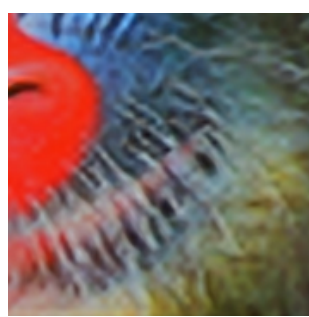

SRCNN

(22.70/5.89)

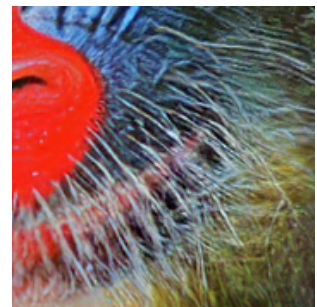

ESRGAN+

(19.79/1.81)

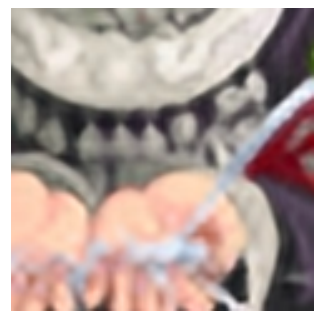

SRCNN

(22.52/6.74)

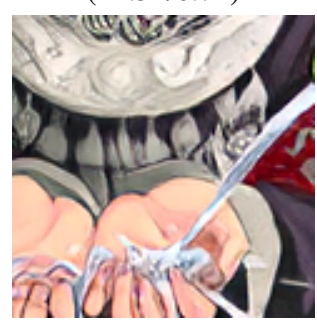

ESRGAN+

(18.06/2.17)

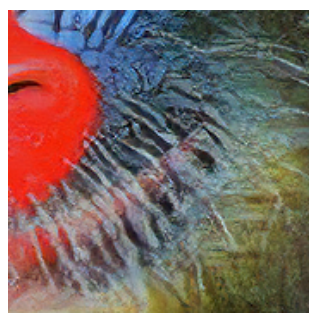

EnhanceNet

(20.87/2.65)

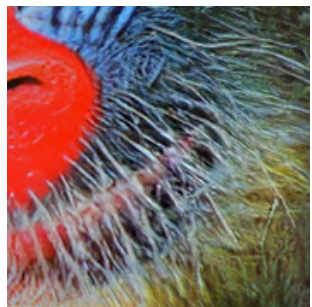

nESRGAN+

(19.71/2.14)

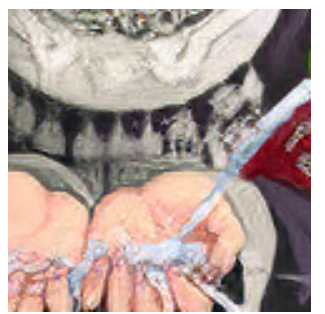

EnhanceNet

(20.64/2.40)

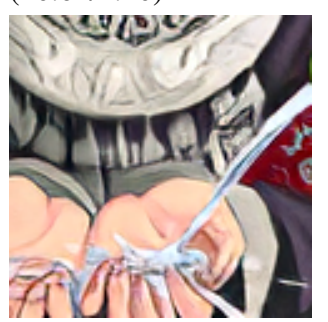

nESRGAN+

(17.76/2.63)

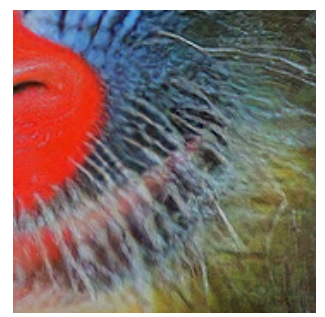

SRGAN

(21.14/2.61)

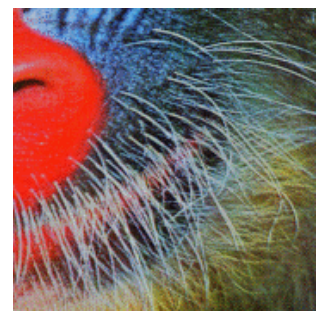

HR

$(\infty / 3.59)$

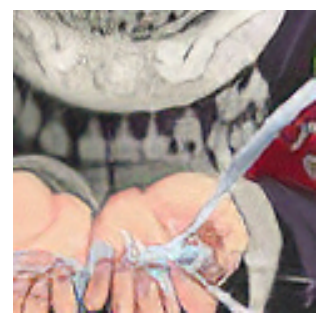

SRGAN

$(19.32 / 2.32)$

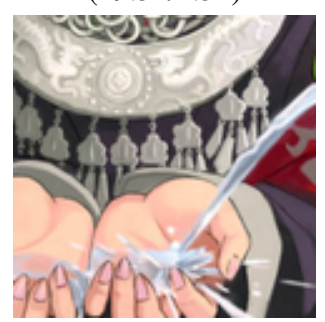

HR

$(\infty / 2.76)$

Fig. 4: Comparison between the qualitative results of the main perceptual-driven models and ESRGAN+ using images from Set14. PNSR (value on the left) and perceptual index (value on the right) are used for the evaluation. 
Table 1: Quantitative evaluation of our models with other perceptual-driven methods. The best and second best results are highlighted and underlined, respectively. We evaluate using the perceptual index (value on the right) but PSNR (value on the left) is also given for reference purposes.

\begin{tabular}{|l|l|l|l|l|}
\cline { 2 - 5 } \multicolumn{1}{c|}{} & EnhanceNet & ESRGAN & ESRGAN+ (ours) & nESRGAN+ (ours) \\
\hline Validation PIRM & $25.06 / 2.68$ & $25.17 / 2.55$ & $24 / 2.38$ & $24.32 / \mathbf{2 . 3 6}$ \\
Test PIRM & $24.94 / 2.72$ & $25.03 / 2.43$ & $23.80 / 2.31$ & $24.15 / 2.29$ \\
Urban100 & $23.54 / \mathbf{3 . 4 7}$ & $24.36 / 3.77$ & $23.28 / \overline{3.55}$ & $23.22 / 3.55$ \\
OST300 & $24.37 / 2.82$ & $24.64 / 2.49$ & $23.84 / \mathbf{2 . 4 6}$ & $23.80 / 2.49$ \\
\hline
\end{tabular}

A PSNR-oriented pre-trained model is used to initialize the generator. The loss function remains unchanged with $\lambda=$ $5 \times 10^{-3}$ and $\eta=1 \times 10^{-2}$. The learning rate is set to $1 \times 10^{-4}$ and halved at $[50 \mathrm{k}, 100 \mathrm{k}, 200 \mathrm{k}, 300 \mathrm{k}]$ iterations.

The model is optimized using Adam with $\beta_{1}=0.9$ and $\beta_{2}=0.999$. The trained model is the one with the 23 blocks generator. The implementation is done with Pytorch and the training with NVIDIA Tesla K80 GPUs.

\subsection{Results}

We evaluate our two models with other perceptual-driven approaches on the PIRM datasets (see Table 1). In the YCbCr color space, PSNR is measured on the luminance channel. The perceptual index is the one used in the PIRM-SR Challenge [26]. It is based on the Ma's score [27] and NIQE [28] and equals $\frac{1}{2}((10-M a)+N I Q E)$. Higher is better when measuring with the PSNR whereas lower is better when considering the perceptual index. Both of our models always perform better compared to ESRGAN. We see that nESRGAN+ has a better perceptual score on the PIRM datasets. This highlights the benefits of using the noise inputs in the generator network. However, there are still limitations associated with the noise injection's generalization. Adding noise does not always result in better perceptual quality. This is the case for categories of images which do not fully exploit stochastic variation such as images of buildings in Urban100 and OST300. Future works will focus on getting the most out of the Gaussian noise.

Qualitative comparison is made in Figure 4 between our models and others based on PSNR and perceptual quality such as SRCNN, EnhanceNet, SRGAN, ESRGAN using images from the Set14 dataset. It can be observed that the images reconstructed by our models present more detailed structures and are less distinguishable from the ground truth images when compared to the other pictures. Most of the original textures are kept like the boy's complexion.

\section{CONCLUSION}

We have proposed ESRGAN+ and nESRGAN+ which outperform other approaches as long as perceptual quality is concerned. A new basic block has been introduced to further increase the capacity of the network. Moreover, noise inputs are added to benefit from stochastic variation. All these improvements have contributed to the generation of images with more natural textures as well as greater sharpness and details.

\section{REFERENCES}

[1] Christian Ledig, Lucas Theis, Ferenc Huszár, Jose Caballero, Andrew Cunningham, Alejandro Acosta, Andrew Aitken, Alykhan Tejani, Johannes Totz, Zehan Wang, et al., "Photo-realistic single image superresolution using a generative adversarial network," in Proceedings of the IEEE conference on computer vision and pattern recognition, 2017, pp. 4681-4690.

[2] Justin Johnson, Alexandre Alahi, and Li Fei-Fei, "Perceptual losses for real-time style transfer and superresolution," in European conference on computer vision. Springer, 2016, pp. 694-711.

[3] Joan Bruna, Pablo Sprechmann, and Yann LeCun, "Super-resolution with deep convolutional sufficient statistics," arXiv preprint arXiv:1511.05666, 2015.

[4] Ian Goodfellow, Jean Pouget-Abadie, Mehdi Mirza, Bing Xu, David Warde-Farley, Sherjil Ozair, Aaron Courville, and Yoshua Bengio, "Generative adversarial nets," in Advances in neural information processing systems, 2014, pp. 2672-2680.

[5] Xintao Wang, Ke Yu, Shixiang Wu, Jinjin Gu, Yihao Liu, Chao Dong, Yu Qiao, and Chen Change Loy, "Esrgan: Enhanced super-resolution generative adversarial networks," in The European Conference on Computer Vision Workshops (ECCVW), September 2018.

[6] Sergey Ioffe and Christian Szegedy, "Batch normalization: Accelerating deep network training by reducing internal covariate shift," arXiv preprint arXiv:1502.03167, 2015.

[7] Alexia Jolicoeur-Martineau, "The relativistic discriminator: a key element missing from standard gan," arXiv preprint arXiv:1807.00734, 2018.

[8] Tero Karras, Samuli Laine, and Timo Aila, "A stylebased generator architecture for generative adversarial networks," arXiv preprint arXiv:1812.04948, 2018. 
[9] Wenming Yang, Xuechen Zhang, Yapeng Tian, Wei Wang, and Jing-Hao Xue, "Deep learning for single image super-resolution: A brief review," arXiv preprint arXiv:1808.03344, 2018.

[10] Chao Dong, Chen Change Loy, Kaiming He, and Xiaoou Tang, "Learning a deep convolutional network for image super-resolution," in European conference on computer vision. Springer, 2014, pp. 184-199.

[11] Chao Dong, Chen Change Loy, Kaiming He, and Xiaoou Tang, "Image super-resolution using deep convolutional networks," IEEE transactions on pattern analysis and machine intelligence, vol. 38, no. 2, pp. 295307, 2016.

[12] Jiwon Kim, Jung Kwon Lee, and Kyoung Mu Lee, "Accurate image super-resolution using very deep convolutional networks," in Proceedings of the IEEE conference on computer vision and pattern recognition, 2016, pp. 1646-1654.

[13] Jiwon Kim, Jung Kwon Lee, and Kyoung Mu Lee, "Deeply-recursive convolutional network for image super-resolution," in Proceedings of the IEEE conference on computer vision and pattern recognition, 2016, pp. 1637-1645.

[14] Ying Tai, Jian Yang, and Xiaoming Liu, "Image superresolution via deep recursive residual network," in Proceedings of the IEEE Conference on Computer Vision and Pattern Recognition, 2017, vol. 1, p. 5.

[15] Gao Huang, Zhuang Liu, Laurens Van Der Maaten, and Kilian Q Weinberger, "Densely connected convolutional networks," in Proceedings of the IEEE conference on computer vision and pattern recognition, 2017, pp. 4700-4708.

[16] Tong Tong, Gen Li, Xiejie Liu, and Qinquan Gao, "Image super-resolution using dense skip connections," in Computer Vision (ICCV), 2017 IEEE International Conference on. IEEE, 2017, pp. 4809-4817.

[17] Mehdi SM Sajjadi, Bernhard Scholkopf, and Michael Hirsch, "Enhancenet: Single image super-resolution through automated texture synthesis," in Proceedings of the IEEE International Conference on Computer Vision, 2017, pp. 4491-4500.

[18] Christian Szegedy, Sergey Ioffe, Vincent Vanhoucke, and Alexander A Alemi, "Inception-v4, inceptionresnet and the impact of residual connections on learning," in Thirty-First AAAI Conference on Artificial Intelligence, 2017.

[19] Yunpeng Chen, Jianan Li, Huaxin Xiao, Xiaojie Jin, Shuicheng Yan, and Jiashi Feng, "Dual path networks," in Advances in Neural Information Processing Systems, 2017, pp. 4467-4475.

[20] Eirikur Agustsson and Radu Timofte, "Ntire 2017 challenge on single image super-resolution: Dataset and study," in Proceedings of the IEEE Conference on Computer Vision and Pattern Recognition Workshops, 2017, pp. 126-135.

[21] David Martin, Charless Fowlkes, Doron Tal, Jitendra Malik, et al., "A database of human segmented natural images and its application to evaluating segmentation algorithms and measuring ecological statistics," Iccv Vancouver:, 2001.

[22] Jia-Bin Huang, Abhishek Singh, and Narendra Ahuja, "Single image super-resolution from transformed selfexemplars," in Proceedings of the IEEE Conference on Computer Vision and Pattern Recognition, 2015, pp. 5197-5206.

[23] Xintao Wang, Ke Yu, Chao Dong, and Chen Change Loy, "Recovering realistic texture in image superresolution by deep spatial feature transform," in The IEEE Conference on Computer Vision and Pattern Recognition (CVPR), June 2018.

[24] Marco Bevilacqua, Aline Roumy, Christine Guillemot, and Marie Line Alberi-Morel, "Low-complexity singleimage super-resolution based on nonnegative neighbor embedding," 2012.

[25] Roman Zeyde, Michael Elad, and Matan Protter, "On single image scale-up using sparse-representations," in International conference on curves and surfaces. Springer, 2010, pp. 711-730.

[26] Yochai Blau, Roey Mechrez, Radu Timofte, Tomer Michaeli, and Lihi Zelnik-Manor, "The 2018 pirm challenge on perceptual image super-resolution," in Proceedings of the European Conference on Computer Vision (ECCV), 2018, pp. 0-0.

[27] Chao Ma, Chih-Yuan Yang, Xiaokang Yang, and MingHsuan Yang, "Learning a no-reference quality metric for single-image super-resolution," Computer Vision and Image Understanding, vol. 158, pp. 1-16, 2017.

[28] Anish Mittal, Rajiv Soundararajan, and Alan C Bovik, "Making a "completely blind" image quality analyzer," IEEE Signal Processing Letters, vol. 20, no. 3, pp. 209212, 2012. 